\title{
XVII.
}

\section{Ueber den Nutzen der pancreatischen Flüssigkeit bei der Verdauung.}

\author{
Von \\ Ch. Bernaral. \\ (Annal. de chim. et de phys. XXV, 47t)
}

Seit langer Zeit betracliten die Anatomen das Pancreas als eine Speicheldrüse des Bauches. Olıne Zweifel durch dieselbe Induktion und auf nicht genügende Versuche gestintzt, habon in der jüngsten Zeit einige Physiologon der pancreatischen Flüssigkeit die Eigenschaften des Speichels zugeschrieben. Diese Vergleichung des Pancreas und der Speicheldrūsen ist aber falsch Innd sie erklärt keineswegs den Nutzen der pancreatischen Flüssigkeit bei den Verdauungserscheinungen.

In nachstehender Abhandlung nahm ich mir vor, auf experimentellem Wege nachzuweisen, dass die pancreatische Fliissigkeit, ausschliesslich aller anderen Eingetouideflüssigkeiten, die Bestimmung hat, die neutralen, in den Vahrungsmilteln enthallenen fetten Substansen su nerdalen und dieselben auf diese Weise ₹ur Absorption durch die Saugallern geschickt su machen.

Ehe ich in das Detail meiner Untersuchungen eingehe, will ich die verschiedenen Umstände angeben, welche die Eigenschaften der pancreatischen Flüssigkeit modificiren köınun, damit die später anzuführenden Resultate deutlicher werden und leichter wiederholt werden künnen.

\section{Ueber die Ausscheiluny der panureatischen Ftüssigkeit und uber die Bedingungen der Secretion derselben.}

Bis jetzt habe ich die pancreatische Flüssigkeit von vier und dreissig Hunden ausgeschieden. Das zu diesem Zweck angewendete Verfahren ist das zuerst von $\mathrm{Magendie,} \mathrm{dann} \mathrm{von}$ Tiedeman $n$ und $\mathbf{G m e l i n}$ benutzte, welches wesentlich darin besteht, in das Hypochondrium rechts unter den Rippen einen Einschnitt zu machen, um das Duodenum und einen Theil des Pancreas bloss zu legen. Darauf isolirt man, so schnell als 
möglich, den dicksten der beiden pancreatischen Gänge und hefestigt in demselben ein silbernes Rölirchen; nachdem dies geschehen, bringt man das Pancreas und Duodenum in das Abdomen zurück, und näht die Wunde auf die Weise zu, dass das Ende der silbernen Röhre aussen befindlich ist, damit die pancrealische Flüssigkeit durch dieselbe abgelassen werden kann. An das Ende der Röhre befestigt man eine kleine, Kautschukflasche, aus welcher vorher durch Zusammendrücken die Luft entfernt worden ist; indem diese Flasche ihre vorige Form wieder anzunehmen strebt, saugt sie die Flüssigkeit über. Dieser Apparat hält gewöhnlich zwei oder drei Tage; nach dieser Zeit fallt er mit dem Verbande ab. Die Hunde sterben niemals an dieser Operation, wenn sie geschickt gemacht worden ist. Die Wunde vernarbt leicht und der pancreatische Gang regenerirt sich sehr schnell.

Bei strenger Befolgung dieses Verfalırens habe ich bemerkt, dass die Menge der erhaltenen pancreatischen Flüssigkeit um se grōsser ist, je schneller die Operation vor sich ging und je kürzere Zeit das Pancreas der Luft ausgesetzt war. Unabluängig aber von der Operationsmethode muss man noch die andern Bedingungen kennen. So geht während der Verdauung die Secretion der pancreatischen, Flüssigkeit am reichlichsten vor sich, und zu dieser Zeit kann man ungefähr zwei Grammen stündlich von einem Hunde mittlerer St:tur erhalten. Wäbrend des Fastens hingegen ist die Secretion des pancreatischen Saftes fast null und die Menge der erhaltenen Flüssigheit unbedeutend und zu irgend einer Untersuchung nicht hinreichend. Zu diesen beiden Perioden bietet übrigens das Pancreas ein wesentlich verschiedenes dussehen dar. Während des Fastens ist das Gewebe dieses Organes blass und milchig-weiss, umgekehrt wăhrend' der Verdauung ist es roth und von Blute strotzend.

Ausser diesen Umständen, welche auf die Quantităt der pancreatischen Flüssigkeit von Einflusse sind, giebt es noch deren, die noch weit wichtiger sind, weil sie die Qualität dieser Flüssigkeit betreffen. Bei Befolgung des angegebenen Verfahrens ereignet es sich, dass einige Zeit nach der Operation das Thier vom Fieber ergriffen wird und das Pancreas und die Bauchwunde krank werden. Es findet dahei das Eigentbüu- 
liche stalt, dass die Menge der pancreatischen Flüssigkeit sich nicht vermindert, sondern beträchtlich zunimmt. Diese krankhafte pancreatische Flüssigkeit ist aber, obgleich sie noch alkalisch reagirt, nicht mehr klebrig und hat alle charakteristischen Eigenschaften verloren.

Diese Veränderung der Flūssigkeit in Folge des Krankseins des Prancreas erfolgt in unbestimmter Zeit, gewöhnlich zwar erst den folgenden Tag. zuweilen aber auch kurze Zeit nach der Operation, zumal wenn dieselbe schwierig von statten ging und die Baucheingeweide längere Zeit der Luft ausgesetzt waren.

In Bezug auf die Beschaffenheit muss man deshalb zwei Arten der pancreatischen Flüssigkeit unterscheiden: 1) diejenige, die wir normal nennen, und die sogleich nach der Operation, ehe das Pancreas erkrankt, ausgeschieden wurde; 2) die krankhafte, welche ausgeschieden wird, wenn sich in dem Pancreas und der Bauchwunde die Symptome des Wundfiebers zeigen. Dieser Unterschied ist nicht nur für das Studium der physischen und chemischen Eigenschaften der pancreatischen Flüssigkeit von Wichtigkeit, sondern er ist auch unerlässlich, wenn man die physiologischen Eigenschanten dieser Flüssigkeit bei der Verdauung erklären will.

\section{Physikalische und chemische Charactere der pancreatischen \\ Flüssigktir.}

l)ie normale, unter günstigen Bedingungen erhaltene pancreatische Flüssigkeit ist ein farbloses helles, schleimiges und klebriges Liquidum, das in grossen, perlartigen, syrupähnlichen Tropfen đliesst, unł beim Schủtteln schäumt. Diese Flüssigkeit ist ohne eigenthümlichen Geruch; auf die Zunge gebracht, erregt sie das Gefühl einer schmierigen Flüssigkeit, ihr Geschmack hat elwas salziges und ist dem des Blutserums nicht unähnlich. Eben so wie Mage ndie und andere Beobachter, fand ich die pancreatische Flüssigkeit deutlich alkalisch gegen lakmuspapier reagiren. Ich fand sie nie sauer oder neutral. Die normale pancreatische Flüssigkeit coagulirt in der Wärme und verwandelt sich in eine weisse feste Masse. Die Coagulation ist dieselbe wie beim Eiweiss. Die pancreatische Flüssigkeit wird ebenfalls durch Salpetersäure, Schwefelsäure und concentrirte Chlorwasserstoffsăure gefältt. Metallsalze, Holzgeist und Alkohol coaguliren 
gleichfalls die organische Substanz der pancreatischen Flüssigkeit. Verdünnte Essigsāure, Milchsāure und verdūnnte Chlorwasserstoffsảure bewirken keine Coagulation. Die Alkalien erzeugen keinen Niederschlag und lösen selbst den durch Wärme, Säuren und Alkohol entstandenen wieder auf.

Ich fand, dass die pancreatische Flüssigkeit des Pferdes, Seidenhasens, der Vögel (Tauben und Hühner), wenn sie unter günstigen Bedingungen erhalten wurde, eben so wie die des Hundes eine mehr oder weniger fadenziehende Flūssigkeit ist, die deutlich alkalisch reagirt und durch Erhitzen vollstāndig coagulirt wird.

Stellt man die Charactere der pancreatischen Flüssigkeit zusammen, so kann man daraus den Schluss ziehen, dass dieselbe sich gegen Reagentien wie eine eiweisshallige Flüssigkeit verhălt. Denn eine lösliche Substanz, die durch Erhitzen und durch Säuren coagulirt wird, hat Eigenschaften, die denen des Albumins gleich sind. In physiologischer Beziehung aber findet zwischen der pancreatischen Flüssigkeit und einer eiweissartigen Substanz, wie wir sogleich sehen werden, keine Analogie statt. Ich will zu beweisen suchen, dass dieses coagulirbare Princip der wirksame Stoff bei der Verdaunng ist, und dass die organische Substanz der pancreatischen Flüssigkeit physiologische Eigenschaften zeigt, welche dem Albumin nicht zukommen. Es gelang mir, Unterscheidungskennzeiclsen zwischen dieser Substanz und dem Eiweiss aufufinden. Ich erwähne nur das folgende, das mir entscheidend zu sein scheint.

Wenn die Substanz des pancreatischen Saftes mittelst absolutem Alkohol coagulirt und bei gelinder Wärme getrocknet worden ist, so losst sie sich leicht und vollständig in Wasser, während auf gleiche Weise behandeltes Albumin sich nicht mehr löst. Ich bemerke noch, dass bei der Auflösung die pancreatische Substanz dem Wasser das eigenthümlich Klebrige und alle physiologischen Eigenschaften des pancreatischen Saftes ertheilt, so dass man nicht langer daran zweifeln kann, dass diese Substanz wirklich das wirksame Princip in dieser Flüssigkeit sei.

Krankbafte oder veränderte pancreatische Flüssigkeit ist ein Liquidum von der Consistenz des Wassers, ohne alle Klebrigkeit, gewühnlich farblos, of aber auch opalisirend und zuweilen 
106 Bernard: Ueber den Nutzender pancreatischen

sogar röthlich gefärbt. Diese Flūssigkeit ist von salzigem und zugleich ekelerregendem Geschmacke. Ihre Reaction ist stets stark alkalisch; sie wird aber weder durch Erhitzen, noch durch Säuren coagulirt und unterscheidet sich dadurch wesentlich vom normaleu pancreatischen Safte.

\section{Physiologische Eigenschaften der pancreatischen Elüssig- keil ausserhalb des Oryanismus.}

Im Anfange dieser Abhandlung sagte ich, dass die pancreatische Flüssigkeit, ausschliesslich aller anderen Eingeweidenussigkeiten, die Bestimnsung habe, auf eigenthümliche Weise die sich in den Nahrungsmitteln vorfindenden fetten Substanzen zil verändern. Dies lässt sich Jeicht nachweisen, wenn man in einer Röhre pancreatische Flüssigkeit auf fette Körper einwirken lässt.

Mengt man bei einer Temperatur von $38-40^{\circ}$ parcreatische Flüssigkeit mit Oel, Bulter oder Fett, so bemerkt man, dass die fette Substanz augenblicklich in eine vollständige Emulsion verwandelt wird. Es entsteht eine weisse, rahmartige Flüssigkeit, die dem Cbylus vollkommen gleicht. Prüft man die Charaktere dieser Emulsion näher, so findet man, dass unter dem Einflusse der pancreatischen Flüssigkeit die fette Substanz nicht nur zertheilt, sondern auch chemisch verāndert worden ist. In der That besitzt im Augenblicke des Mengens der fetten neutraJen Substanz mit der alkalischen pancreatischen Flüssigkeit das Gemenge eine deulliche, alkalische Reaction, die bald nachher in eine eutschieden saure ubergeht. Ich prüfte im Laboratorium von Pelouze, in Verein mit Barreswil und Margueritte diese Produtste; wir fanden, dass sich das Fett in Fettsiure und und in Glycerin zersetzt hatte. Wählt man die Butter, so bemerkt man bald die Buttersãure an ihrem charakteristischen Geruche.

Aus Vorstehendeln folgt deutlich, das der pancreatische Saft, ausserhalb des Organismus die Eigenschaft besitst, neutrale folte Körper rollsländig und augenblicklich in felte Säuren und in Glyeerin zu verwandeln.

Die pancreatische Flüssigkeit hat unter allen anderen Flüssigkeiten des Organismus allein diese Eigenschaft. Ich versuchte vergleichungsweise die Einwirkung der Galle, des Speichels, des 
gastrischen Saftes, des Blutserums und des Gehirnwassers auf die Fettsubstanz; ich konnte aber weder Emulsion noch irgend eine Veränderung des Fettes bemerken.

Vorstehende Resultate sind so of erbalten worden, und die Versuche sind so kliar und so einfach zu wiederholen, dass jeder sich leicht ron der Wahrheit überzeugen kann.

Es ist hier Gelegenheit, einen wesentlichen Unterschied zwisclien der normalen und der kranklıaften odur veränderten pancreatischen Flüssigkeit anzuführen. In der That wird die augenblickliclıe Vertheilung der neutralen Fettsubstanzen und ilır Zerfalleu in Glycerin und fette Säuren, nur durch den normalen pancreatischen Saft, d. I. durch den klebrigen, durch die Hitze und Säuren coagulirenden pancreatischen Saft bewirkt. Mengt man umgekehrt 0 el oder Felt mit krankhafter oder veränderter pancreatischer Flüssigkeit, so findet keine Einwirkung auf die fetten Substanzen statt und die unthätige pancreatische Flūssigkeit scheidet sich bald von dem unveränderten Fette ab.

\section{Eigenschaften der pancrealischen Flüssigkeit in dem lebenden Thiere benbachtet.}

Nach dem Vorstehenden sollte man glauben, dass bei den lebenden Thieren, die pancreatische Flüssigkeit stets normal sein müsste, und dass deshalb ibre specifische Einwirkung auf die neutralen Fettsubstanzen leicht zu constatiren wäre. In der That folgt aus nachstehenden Untersuchungen, dass der pancreatische Saft, indem derselbe die fetten Substanzen zertheilt und verändert, letztere assimilirbar macht; auf diese Weise wird diese Substanz das unerlässliche Agens, durch welches die weisse homogene Flüssigkeit, die in den Milchgefässen circulirt und Chylus genannt wird, sich bildet. Es ist hier nicht der Ort, über die Bedeutung des Wortes Chylus zu discutiren; ich erinnere nur an ein den Physiologen wohlbekanntes Factum, dass nïmlich ' die Milchgefässe nur dann eine weisse, milchige Flüssigkeit enthalten, wenn sie in den Eingeweiden fette Substanzen absorbirt hatten. Auf diese Weise ist der klare und durchsichtige Chylus frei von fetten Kōrpern, während ein weisser, milchiger Chylus fetthaltig ist. Da dieses festgestellt ist, war es leicht zu zeigen, dass die pancreatische Flüssigkeit in den Eingeweiden das Fett zertheilt und es dadurch für die Milchgefãsse' ab- 
sorbirbar macht. Als ich Hunden die beiden pancreatischen Gaange verband, fand ich stets, dass das Fett den Darmkanal passirte, ohne verändert worden zu sein; dabei war der Chylus durchsichtig, farblos und vollständig von jeder Fettsubstanz frei.

Man könnte sich mit diesen Versuchen begnūgen; ich fand aber noch ein anderes Mittel, dasselbe Factum durch einen gewissermassen von der Natur vorbereiteten Versuch $z u$ beweisen, der sehr leicht zu wiederholen ist. Bei dem Kaninchen scheint die Natur die Wünsche des Experimentators gleichsam errathen zu haben, indem bei diesem Thiere der pancreatische Kanal, der einfach ist, sehr tief, 35 Centimeter unter dem Gallengange einmündet. Bringt man etwas Fett in den Magen eines Kaninchens so geschieht es, dass dasselbe in Berührung mit dem gastrischen Safte unverăndert bleibt und bis in die Eingeweide gelangt, ohne eine Veränderung zu erleiden. Sobald das Fett aber 35 Centimeter unter der Galle mit dem pancreatischen Safte zusanmen kommt, enthalten die Milchgefässe einen weisslichen, fettigen Chylus, während sie hơher mit farblosem, durchsichtigem Chylus angefüllt sind. Es giebt mithin bei dem Kaninchen unter diesen Umständen zwei Arten von Chylus, 1) den durchsicbtigen und fettfreien, der von den Eingeweiden, die 35 Centimeter üher der Mündung des pancreatischen Canales liegen, herrübrt und 2) den weissen, homogenen, fetthaltigen, der unterhalb der Mündun: des pancreatiscben kanales entstanden ist.

Der Anblick der Milchgefasse eines Kaninchens zeigt auf einfache und entscheidende Weise, dass die Flüssigkeit, welche man mit dem Namen Chylus bezeichnet, nicht ohne die Mitwirkung der pancreatischen Flüssigkeit gebildet werden kann.

\section{Schlüsse.}

Ich glaube auf experimentellem Wege bewiesen zu haben, dass die pancreatische Flüssigkeit die Bestimmung hat, die neutralen, fetten, zur Nahrung dienenden Substanzen zu verdauen und auf diese Weise, ibre Absorplion durch die Milchgefásse zu gestatten. Ich glaube zur Unterstützung meiner Behauptung hinreichende Experimente angestellt zu baben; es sei mir jedoch gestatteh einige Thatsachen anzuführen, die mit dem Gesagten in Widerspruch zu stehen scheinen. 
Brodie fand im Jahre 1823, dass, als er den Gallengang einer Katze unterbunden hatte, das Fett in dem Darmkanal nicht mehr in eine Emulsion verwandelt war und dass der Chylus dieser Thiere klar und von jeder Fettart frei war. Daraus schloss er, dass die Galle es sei, welche die fetten Substanzen in Emulsion verwandele und sie absorbirbar mache. Magendie wollte diesen Versuch bei Hunden wiederholen und fand, den Beobachtungen Brodie's entgegen, dass ungeachtet der Abwesenheit der Galle in dem Darmkanal, das Fett in Emulsion verwandelt war und dass die Milchgefässe der Hunde einen weissen, milchigen, felthaltigen Clyylus enthielten. Diese verschiedenen Resultate lassen sich jetzt sehr leicht erklären. Bei der Katze findet zwischen dem Gallengang und dem pancreatischen Gange Anastomose statt, ehe heide in den Darmkanal mūnden, so dass auf diese Weise, da die Mündung der beiden vereinigten Kanäle verhindert wurde, auch die Einwirkung der pancreatischen Flüssigkeit auf das Fett nicht stattinden konnte. Bei dem Schlusse schrieb der Experimentator der Galle zu, was in der That der Flüssigkeit des Pancreas zugeschrieben werden musste.

II agendie stellle sein!: Versuche mit Hunden an, bei welchen der Gallengang im Gegentheil vollständig von den beiden pancreatischen Gúngen isolirt ist. Da durch die Operation das Ausfliessen der pancreatischen Flüssigkeit nicht verhindert worden war, so musste das Fett in eine Emulsion verwandelt werden und der Chylus weiss und fetthaltig erscheinen.

Diese Versuche sind demnach nicht im Widerspruche; der Unterschied der Resultate erklārt sich durch die eigenthümliche Disposition der pancreatischen Gänge bei den verschiedenen Thierspecies, die zu den Versuchen angewendet wurden. Diese Versuche unterstútzen deshalb beide meine Untersuchungen und beweisen, dass es nur die pancreatische Flüssigkeit und nicht die Galle ist, welche das Fett in Emulsion verwandelt und dasselbe in den Chylus überfübrt.

Schliesslich muss ich noch bemerken, dass meine Untersuchungen die Beobachtungen von Bou chardat und $S$ a ndras, nach welchen die Stärke durch die pancreatische Flüssigkeit in Krümelzucker umgewandelt wird, keineswegs entlkräntigen. In Bezug auf diesen Gegenstand will ich nur erwähnen, dass die Einwirkung der pancreatischen Flüssigkeit auf die Stärke, die 
ich ebenfalls bemerkt habe, dieser Flüssigkeit nicht eigenthumlich ist. Diese Eigenschaft kummt dem Speichel des Menschen und der Thiere, dem Blutserum und allen alkalischen Flüssigkeiten des normalen oder pathologischen Organismus zu. Dies wurde durch die Arbeit von Magendie und Reyer und auch durch meine eigenen Beobachtungen bestätigt. Ich füge linzı, dass die Umwandlung der Stärke in Krümelzucker eben sowolı! unter dem Einflusse der normalen, als auch der kranlilıaften, veränderten pancreatischen Flüssigkeit vor sich geht.

Daraus schliesse ich, dass durch die Umwandlung der Stärke in Krümelzucker die pancreatische Flüssigkeit von keiner andern alkalischen Flüssigkeit des Organismus unterschieden wird, während ihr Vermögen, die neutralen Fettsubstanzen in Emulsion zu verwandeln, ihren wesentlichen und speciellen Character bei der Verdauung ausmacht. Sie theilt diese Eigenschaft mit keiner andern Flüssigkeit der Eingeweide und verliert sie sogleich, sobald die darin enthaltene coagulirbare Substanz rerāndert ist.

\title{
XVIII.
}

\section{Ueber einige den Verdauungsprocess be- treffende quantitative Verhältnisse.}

\author{
Vom \\ Prof. Dr. Lehmann.
}

(Aus d. Ber. über d. Verhandlungen der K. S. Geselischaft der Wissenschaften zu Leipzig. Mathem.-phys. Klasse. 1849. No. I.)

Man hat in neurer Zeit den Process der Magenverdauung oft als schlagendes Beispiel einer rein chemischen Action im lebenden Thierkðrper angeführt: allein leider sind wir noch weit davon entfernt, diesen Process in dem Aufeinanderwirkeu der einzelnen dabei concurrirenden Substrate und den Resultaten der Wirkungen und Gegenwirkungen wissenschaflich erforscht $2 u$ haben. Das eigentliche Ferment der Verdauung, das wir immerhin Pepsin nennen konnen, ist noch wenig untersucht, ja viele zweifeln noch an dessen Existenz oder Wirkungsfähig- 